\title{
BLADDER CALCULI TWO DIFFERENT ENDOSCOPIC TECHNIQUES IN ITS MANAGEMENT
}

\section{General Surgery}

\section{ABSTRACT}

Introduction- presenting our experience of comparison of two endoscopic treatments for bladder stones.

Material and Methods - Study includes 45 patients of vesical calculi, treated in our hospital between June 2010 to December 2017, randomly assigned in two groups group 1- transurethral removal using nephroscope and group 2 -percutaneous suprapubic removal using nephroscope.

Results- Significant difference was observed in operating time group $1(32.1+8.5 \mathrm{mins})$ and group $2(46+7.3)$, statistically difference was seen in the post operative stay of patient which was higher in group 2, complete clearance was seen in both groups, while group 1 had maximum number of urethral entries in comparison to supra pubic group

Conclusion- Transurethral vesical stone treatment using nephroscope is effective route of treatment of large vesical stones without increasing morbidity and without giving a supra pubic scar.

\section{KEYWORDS}

\section{INTRODUCTION}

Vesical calculi accounts for $5 \%$ of urinary stones (1), usually symptomatic few can be asymptomatic, classified into Primary-which take origin in kidneys and lodge in bladder and Secondary which are due to bladder outlet obstruction, neurogenic , foreign body, bladder diverticulum, trauma and catheterisation etc.

Surgical treatment evolved from blind insertion of crushing forceps in bladder to open surgery to extracorporeal fragmentation of bladder stone .Open surgery in past was the best recommended modality for large stones(2). Kemal et al. in there study concluded the effectiveness of transurethral stone treatment endoscopically via cystoscope (3).

In this study we present two techniques of bladder stone removal endoscopically ,both using nephroscope, via transurethral route (natural) and percutaneously ( supra pubic) route.

\section{MATERIALAND METHODS}

The study had 45 patients of bladder stone treated at MMC ,Muzaffarnagar ,Gen.Surgery department, by a single urologist ,between June 2010 to December 2017, after consent ,patients were randomised in two groups group 1 (transurethral removal using nephroscope) and group 2 (percutaneous removal using nephroscope). Pre-operative evaluation had history, physical examination ,routine labs ,X'ray KUB and Ultrasound abdomen

After Spinal Anaesthesia, cystoscopy was performed using karl storz $20 \mathrm{fr}$. cystoscope to determine size, number of stone and any associated pathology in bladder and urethra. Pneumatic energy was used to fragment the stone in both groups.

In group 1- 24 fr rigid nephroscope sheath (karl storz) was introduced in bladder via urethra with a blind obturator ,later obturator was removed and nephroscope was passed in sheath, stone in bladder was visualised and fragmented with pneumatic energy into small fragments, all the fragments later were retrieved using ellick evacuator, after complete stone clearance $16 \mathrm{fr}$ foleys catheter was placed in bladder. Patient was discharged the next day after catheter removal.

In group 2- After cystoscopy suprapubic puncture was made using puncture needle, guide wire was passed in bladder and tract dilated upto $26 \mathrm{fr}$, Amplatz sheath secured over dilators in bladder suprapubically, entire process was done using $24 \mathrm{fr}$ karl storz nephroscope, stones fragmented with pneumatic energy and retrieved using forceps via nephroscope .After completion of procedure a suprapubic catheter was placed via suprapubic tract (removed next day), and a urethral catheter was placed which was removed second post op day, patient was sent home after two days.

Eleven patients ( group $1=5$, group $2=6$, required additional procedures- optical internal urethrotomy, transurethral resection of prostate, meatotomy and bladder neck incision, at the time of treatment , the operative time for these additional procedures were not included in the operative time used for statistical analysis.

Antibiotics were given during stay, per operative and for 10 days post operatively, X'ray KUB done post op to confirm stone free status.

Two patients requiring open stone surgery were excluded from this study.

Statistical analysis was performed using Mann-Whitney U test and difference were accepted to statistically significant at $\mathrm{p}<0.05$. The groups were compared of age ,stone size ,operative time, clearance of stone and duration of postoperative stay.

\section{RESULTS}

45 patients of bladder stone were treated between June 2010 to December 2017, only two patients requiring cystolithotomy , and were excluded from this study as this procedure is seldom done these days and the number of patients was less and would have caused skewing of data.

No statistical significance was found in both groups regarding age stone size and number of patients (TABLE 1). Statistical significant difference was observed in operating time : group $1(32.1+/-8.5 \mathrm{~min})$ and group 2(46+/-7.3). Statistically significant difference was observed in the post operative stay of the patients in group 2, complete clearance was achieved in all cases group 1 had slightly more urethral entries as compared to other group.

The operative time for additional procedures was not included in analysis. The patients of bladder neck contracture and stricture urethra were advised regular calibration of urethra according to standard protocol follow up

Table 1 : Comparison of two groups for various variables

\begin{tabular}{|l|l|l|}
\hline & Group 1 & Group 2 \\
\hline No. of patients & 22 & 23 \\
\hline Age (yrs+SD) & $45.6+11.2$ & $47.6+10.8$ \\
\hline Stone size (cms+SD) & $2.9 \mathrm{cms}+1.1$ & $3.1+1.2$ \\
\hline Operative time (mins) & $32.1+8.5 \mathrm{mins}$ & $46+7.3 \mathrm{mins}$ \\
\hline Additional procedure & 5 & 6 \\
\hline TURP & 1 & 2 \\
\hline OIU & 4 & 3 \\
\hline BNI & 0 & 1 \\
\hline Mean urethral entries & $3.2+0.6$ & $1.04+0.04$ \\
\hline Post operative stay & $1.4+0.6$ & $2.1+0.15$ \\
\hline
\end{tabular}

\section{DISCUSSION}

Variety of treatment modalities are described for removal of bladder stones - open surgical, lithotripsy (ESWL), Percutaneous route and Transurethral route (1-6). All endoscopic procedures aim to achieve stone free status in shortest time with short hospital stay and minimal complications. Pneumatic lithotripter is widely used in most of the urological centres and is cost effective method in stone fragmentation. 
The advantage of nephroscope is better vision and the pneumatic probe is more sturdier.

Percutaneous removal of bladder stone is not a new procedure, placement of suprapubic amplatz sheath helps in better visualisation, fragmentation and also prevents prolonged instrumentation of urethra which can lead to stricture urethra. Ahmadina et al.in their study found this modality to be more beneficial in treating bladder stones in children (7).Percutaneous approach is preferred in children due to narrow urethra and its use may cause stricture urethra(8), it is also used to treat stones in artificially created bladder (9-10). Over the years, there is a increasing trend to use nephroscope to treat bladder stones both urethrally and suprapubically .

No patient in the urethral group developed stricture on follow up ,the reason for which would be short intra urethral time of surgery due to 24 fr. sheath, the surgery had less time and the inflammatory reaction of urethra by catheter was less as in transurethral group the urethral catheter was removed the next day and in suprapubic group on second day ( one day later).

\section{CONCLUSION}

Transurethral stone removal using a nephroscope is as safe and effective for stone removal as suprapubic route ,the shorter surgical time in using urethral route favours near negligible injury to urethra, and urethral route can be used in management of big bladder stones with success, and saves the patient from a suprapubic scar which is made in per cutaneous approach.

\section{REFERENCES}

1. Schwartz BF,Stoller ML. The Vesical calculus Urol Clin North America 2000;27:333-46

2. Maheshwari PN,Oswal AT, Bansal M.Percutaneous cystolithotomy for vesical calculi a better approach Tech Urol 1999;5:40-2er 1999;84:332-6

3. Asci R,Aybek Z Sarikaya S,Buyukalpelli,Yilmaz AF :The management of vesical calculi with optical mechanical cystolithotripsy and transurethral prostatectomy is it safe and effective? BJU int

4. Zhaowu Z,Xiwen ,Fenling Z. Experience with Electrohydraulic shock wave in treatment of vesical calculi.Br J Urol1988;61:498-500

5. Ener K,Agras K, Aldemir M,Okulu E,Kayigil O. The randomised comparison of two different endoscopic techniques in the management of large bladder stones :transurethral use of nephroscope or cystoscope ? J Endourol 2009;23:1151-5

6. Aron M,Goel R, Gautam G,Seth A,Gupta NP.Percutaneous versus transurethral cystolithotripsy and TURP for large prostates and large vesical calculi :refinement of technique and updated data. Int UrolNephrol 2007;39:172-7

7. Sathaye UV .Per-urethral endoscopic management of bladder stones :does size matter? Sathaye UV .Per-urethral endoscopic management of bladder stones :does size matter ?
Endourol 2003;17:511-2

8. Ahmadnia H,Younesi Rostami M,Yarmohammadi AA,Parizadeh SM,Esmaeili M,Movarekh M. percutaneous treatment of bladder calculi in children 5 years experience.Urol J 2006;3:20-2

9. Miller DC,Park JM. Percutaneous cystolitotomy using a laproscopic entrapment sac. Urology 2003;62:333-6

10. Cain MP ,Castle AJ,Kaefer M,Yerkes E,Rink RC .Percutaneous cystolithotomy in the pediatric augmented bladder J Urol 2002;168:1881-2 